\title{
Downregulation of miR-542-3p promotes osteogenic transition of vascular smooth muscle cells in the aging rat by targeting BMP7
}

\author{
Huan Liu ${ }^{2,3+}$, Hongwei Wang ${ }^{5 \dagger}$, Sijin Yang ${ }^{4^{*}}$ and Dehui Qian ${ }^{1^{*}}$
}

\begin{abstract}
Background: Aging is believed to have a close association with cardiovascular diseases, resulting in various pathological alterations in blood vessels, including vascular cell phenotypic shifts. In aging vessels, the microRNA(miRNA)mediated mechanism regulating the vascular smooth muscle cell (VSMC) phenotype remains unclarified. MiRNA microarray was used to compare the expressions of miRNAs in VSMCs from old rats (oVSMCs) and young rats (yVSMCs). Quantitative reverse transcription real-time PCR (qRT-PCR) and small RNA transfection were used to explore the miR-542-3p expression in OVSMCS and YVSMCS in vitro. Calcification induction of YVSMCs was conducted by the treatment of $\beta$-glycerophosphate ( $\beta$-GP). Alizarin red staining was used to detect calcium deposition. Western blot and qRT-PCR were used to investigate the expression of the smooth muscle markers, smooth muscle 22a (SM22a) and calponin, and the osteogenic markers, osteopontin (OPN), and runt-related transcription factor 2 (Runx2). Lentivirus was used to overexpress miR-542-3p and bone morphogenetic protein 7 (BMP7) in yVMSCs. Luciferase reporter assay was conducted to identify the target of miR-542-3p.

Results: Compared with yVSMCs, 28 downregulated and 34 upregulated miRNAs were identified in oVSMCs. It was confirmed by qRT-PCR that oVSMC expressed four times lower miR-542-3p than yVSMCs. Overexpressing miR-542-3p in yVSMCs suppressed the osteogenic differentiation induced by $\beta$-GP. Moreover, miR-542-3p targets BMP7 and overexpressing BMP7 in miR-542-3p-expressing yVSMCs reverses miR-542-3p's inhibition of osteogenic differentiation.
\end{abstract}

Conclusions: miR-542-3p regulates osteogenic differentiation of VSMCs through targeting BMP7, suggesting that the downregulation of miR-542-3p in oVSMCs plays a crucial role in osteogenic transition in the aging rat.

Keywords: Mir-542-3p, Vascular smooth muscle cells, Osteogenic differentiation, Aging

\section{Background}

Aging is believed to have a close association with cardiovascular diseases [24]. In elderly patients, the outcomes of vascular angioplasty and stenting are poorer than those in younger patients [3] and also with a fairly higher risk of complication [10, 29]. Vascular aging

\footnotetext{
* Correspondence: ysjimn@sina.com; doctoraiandehui@163.com

†Huan Liu and Hongwei Wang contributed equally to this work.

${ }^{4}$ Department of Cardiology and Neurology, The Second Affiliated Hospital of Southwest Medical University, 184 Chunhui Street, Lu Zhou 646000, Sichuan, China

'Department of Cardiology, Second Hospital Affiliated to the Army Medical University, Xinqiao Hospital, Chongqing 400037, China

Full list of author information is available at the end of the article
}

causes various pathological alterations in blood vessels, including chronic inflammation, vascular cell phenotypic shifts, and structural modifications, which lead to remodeling of the vascular wall with increased thickness and stiffness and drive arteriosclerosis and atherosclerosis [16].

Vascular smooth muscle cells (VSMCs) are contractile cells found in blood vessels and express smooth muscle markers, including smooth muscle $\alpha$-actin (SM $\alpha$-actin), smooth muscle $22 \alpha$ (SM22 $\alpha$ ), and calponin. VSMCs are not terminal differentiated cells, which can switch from the contractile phenotype to the synthetic phenotype in response to local cues and give rise to the development

(C) The Author(s). 2019 Open Access This article is distributed under the terms of the Creative Commons Attribution 4.0 International License (http://creativecommons.org/licenses/by/4.0/), which permits unrestricted use, distribution, and 
of aberrant remodeling of vessels [7]. Increasing evidence indicates that aging is a crucial factor promoting the phenotypic shift of VSMCs and results in agingrelated deterioration of blood vessels $[17,25]$. The dedifferentiation of VSMCs into osteoblast-like cells has been demonstrated to be associated with vascular calcification in the vessel wall [7]. The osteogenic transition of VSMCs is accompanied by the gain of osteogenic markers, such as runt-related transcription factor 2 (Runx2), osteopontin (OPN), and alkaline phosphatase [7]. The triggers of the osteogenic transition of VSMCs have been extensively studied, and many risk factors are recognized, including the loss of calcification inhibitors, senescence, cell death and damage, oxidative stress, mechanical stress, and mitochondrial dysfunction [7]. However, in aging vasculature, the mechanism driving the osteogenic transition of VSMCs remains unclarified.

MicroRNAs (miRNAs) are non-coding, single-stranded RNA molecules and function in RNA silencing and posttranscriptional regulation of gene expression, regulating a number of physiological and pathological processes. Within the cardiovascular system, altered miRNA expression has been found in the blood of patients [2], and certain miRNAs have been found to be involved in various cardiovascular diseases, such as myocardial infarction, arrhythmias, hypertension, and atherosclerosis [33]. In the vascular wall, aging is associated with endothelial dysfunction and a heterogeneous phenotypic shift of VSMCs. A number of miRNAs have been demonstrated to be associated the dysfunction of endothelial cells in aging $[13,23,40]$. However, the mechanism that miRNAs affect phenotypic transition in aging VSMCs is incompletely defined.

In the present study, the expressions of miRNAs in VSMCs from old rats (oVSMCs) and young rats (yVSMCs) were compared using miRNA microarray, and 28 downregulated and 34 upregulated miRNAs were identified in oVSMCs compared with those of the yVSMCs. We focused on miR-542-3p on the basis of microarray detection and demonstrated that miR-542-3p inhibited osteogenic transition of yVSMCs induced by $\beta$-glycerophosphate ( $\beta$-GP) through targeting bone morphogenetic protein 7 (BMP7). Our study suggests that, in aging rats, the decrease in miR542-3p expression promotes the osteogenic transition in VSMCs by targeting BMP7.

\section{Materials and methods \\ Cell culture}

Thoracic aortas of rats were harvested and VSMCs were isolated as previously described [31]. Cells from passage 3-5 were used for the experiments.

\section{Microarray analysis}

Approximate $1.5 \times 10^{5}$ cells were plated into 12-well plates precoated with collagen I (Thermo Fisher
Scientific). After synchronization for $24 \mathrm{~h}$ by serum starvation, VSMCs were cultivated in DMEM supplied with $10 \%$ FBS. After 2 days, total RNA was extracted as previously described [28]. Samples were labeled, hybridized, and scanned (Kang-Chen Bio-tech, Shanghai, China), and images were imported into GenePix Pro 6.0 software for grid alignment and data extraction.

\section{Small RNA transfection}

Approximately $2.5 \times 10^{5}$ VSMCs were plated into 6-well plates. After $24 \mathrm{~h}$, transfection was performed with 50 $\mathrm{nM}$ anti-miR-542-3p (yVSMCs), 50-nM pre-miR-542-3p (oVSMCs), or the associated negative controls, using Lipofectamine RNAiMAX (Invitrogen, Carlsbad, CA, USA). After $12 \mathrm{~h}$, fresh DMEM was added and RNA extraction was performed $24 \mathrm{~h}$ later.

\section{Quantitative reverse transcription real-time PCR}

To quantify the miR-543-3p expression, $1.0 \mu \mathrm{g}$ of total RNA was reverse transcribed using a TaqMan miRNA Reverse Transcription kit (Applied Biosystems, Shanghai, China). miR-542-3p expression was measured using a miR-542-3p TaqMan microRNA assay (Applied Biosystems). The expression of miR-543-3p was quantified using the $2^{-\Delta \Delta \mathrm{Ct}}$ relative quantification method. The relative expression levels of miR-542-3p were determined by normalizing to that of housekeeping gene U6.

To detect the expression of smooth muscle-related genes and calcification-related genes, quantitative realtime PCR (qRT-PCR) was conducted following the reverse transcription. The primer sequences used for SM22 $\alpha$, calponin, OPN, Runx2, and glyceraldehyde-3-phosphate dehydrogenase (GAPDH) are listed in Table 1 . The relative mRNA expressions were determined by normalizing to that of the housekeeping gene GAPDH.

\section{Luciferase reporter construction and luciferase activity assay}

BMP7 mRNA was predicted to be a target of miR-542-3p by the use of TargetScan and Miranda. The 3'-UTR of BMP7 containing the predicted targeting site for miR542-3p was synthesized (BMP7 WT). A mutated sequence was synthesized as well by changing 2 nucleotides (BMP7 MUT). The 3'-UTR of the luciferase reporter containing the BMP7 WT sequence or the BMP7 MUT sequence was inserted into the pmirGLO vector (Promega, Beijing, China). The pre-miR-542-3p was synthesized and inserted in the pSilencer4.1 vector (pSilencer4.1-miR-542-3p, Invitrogen).

For the luciferase assay, about $10^{5}$ HEK293 cells were seeded into 12-well plates. Cells were then cotransfected with BMP7 WT vector or BMP7 MUT vector, and pSilencer4.1-miR-542-3p or pSilencer4.1 empty 
Table 1 Primer sequences for real-time PCR

\begin{tabular}{|c|c|c|c|c|}
\hline Genes & $5^{\prime}-3^{\prime}$ & Primers & $\begin{array}{l}\text { Product } \\
\text { Size (bp) }\end{array}$ & Reference sequence \\
\hline SM 22a & $\begin{array}{l}\text { Forward } \\
\text { Reverse }\end{array}$ & $\begin{array}{l}\text { CCCGCCCTCCATGGTCTTCAAG } \\
\text { GCCAAACTGCCCAAAGCCATTAC }\end{array}$ & 165 & NM_031549.1 \\
\hline Calponin & $\begin{array}{l}\text { Forward } \\
\text { Reverse }\end{array}$ & $\begin{array}{l}\text { CGGGCACCAAGCGGCAGATCT } \\
\text { CCGGGGTCAGGCAGTACTTGGGA }\end{array}$ & 165 & NM_031747.1 \\
\hline OPN & $\begin{array}{l}\text { Forward } \\
\text { Reverse }\end{array}$ & $\begin{array}{l}\text { CATCAGAGCCACGAGTTTCA } \\
\text { TCAGGGCCCAAAACACTATC }\end{array}$ & 274 & NM_012881.2 \\
\hline Runx2 & $\begin{array}{l}\text { Forward } \\
\text { Reverse }\end{array}$ & $\begin{array}{l}\text { CAGACCAGCAGCACTCCATA } \\
\text { CAGCGTCAACACCATCATTC }\end{array}$ & 178 & NM_001278483.1 \\
\hline GAPDH & $\begin{array}{l}\text { Forward } \\
\text { Reverse }\end{array}$ & $\begin{array}{l}\text { AAGTTCAACGGCACAGTCAAGG } \\
\text { CGCCAGTAGACTCCACGACATA }\end{array}$ & 139 & NM_017008.4 \\
\hline
\end{tabular}

vector. Luciferase activity assay was performed after 24 h, using the Dual-Luciferase Reporter System (Promega).

\section{Lentivirus construction and transduction}

Lentivirus was constructed and transduced into yVSMCs to overexpress miR-542-3p or BMP7. Briefly, the coding sequences of target cDNAs were subcloned into the pCDH vector and co-transfected into HEK293T cells with psPAX2 and pMD2.G using Lipofectamine 2000 (Invitrogen). Virus particles were collected from the supernatant $48 \mathrm{~h}$ after transfection and used for the infection of yVSMCs. The infected yVSMCs were incubated in growth medium in the presence of puromycin for stable selection. Cells infected by virus particles generated by empty pCDH vector were used as control.

\section{Calcification induction and Alizarin red staining}

To induce calcification, yVSMCs stably expressing miR542-3p were cultivated in growth medium supplemented with 10-nM $\beta$-GP (Sigma-Aldrich; Merck KGaA, Darmstadt, Germany). After a 7-day incubation, yVSMCs were fixed with $95 \%$ ethanol for $30 \mathrm{~min}$ at room temperature and stained with 1\% Alizarin red (Sigma-Aldrich, Merck $\mathrm{KGaA}$ ) for $15 \mathrm{~min}$. After washing, the calcium deposition was photographed under an inverted phase-contrast microscope (Olympus, Tokyo, Japan). The calcium deposition was quantified by dye elution as previously described [9].

\section{Western blot}

VSMCs lysis was conducted using RIPA lysis buffer (Beyotime, Shanghai, China) supplied with $1 \%$ protease inhibitors. The proteins were separated by electrophoresis and transferred onto PVDF membranes by electroblotting for $3 \mathrm{~h}$ at $150 \mathrm{~mA}$. Then, the membranes were blocked for $1 \mathrm{~h}$ at room temperature in Tris-buffered saline containing $0.1 \%$ Tween 20 (TBST) and 5\% skim milk. The membranes were incubated with primary antibodies against BMP7 (Abcam, Cambridge, MA), SM22 $\alpha$ (Abcam), calponin (Abcam), OPN (Cell Signaling Technology, Danvers, MA, USA), Runx2 (Cell
Signaling Technology), and GAPDH (ZSGB-Bio, Beijing, China), and then the HRP-conjugated secondary antibody (MultiSciences, Beijing, China). Chemiluminescent signals were detected and quantified by densitometry using Quantity One Bioanalysis software (Bio-Rad).

\section{Statistical analysis}

Data were presented as means \pm standard deviation (SD). For the comparison of groups, Tukey's multiple comparisons test was used following ANOVA. $P<0.05$ was considered statistically significant.

\section{Results}

miRNA expression in oVSMCs and yVSMCs

In our experiment, 260 miRNAs were statistically analyzed using miRNA array. Our results indicated 28 downregulated and 34 upregulated miRNAs in oVSMCs when comparing with yVSMCs (Additional file 1: Table S1 and S2). According to the microarray data, miR-542$3 \mathrm{p}$ expression was reduced 14-fold in oVSMCs. Given that the effect of miR-542-3p in VSMC senescence and its relationship with cardiovascular diseases has barely known, we focused on miR-542-3p in this study.

To confirm the miRNA microarray data, qRT-PCR was used to explore the miR-542-3p expression levels in yVSMCs and oVSMCs. We found that miR-542-3p was four times lower expressed in oVSMCs versus that in yVSMCs (Fig. 1a). Furthermore, anti-miR-542-3p transfection into yVSMCs resulted in a significant downregulation of miR-542-3p expression (Fig. 1b), while transfection of pre-miR-542-3p into oVSMCs gave rise to a significant upregulation of miR-542-3p (Fig. 1c). These data suggested that miR-542-3p was abundant in yVSMCs whose expression was decreased within the senescence of VSMCs.

miR-542-3p inhibited osteogenic transition in yVSMCs induced by $\beta-G P$

To explore the effect of miR-542-3p on the osteogenic transition, miR-542-3p was overexpressed in yVSMCs 
A

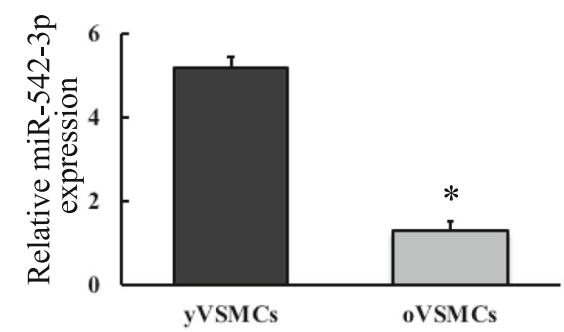

B

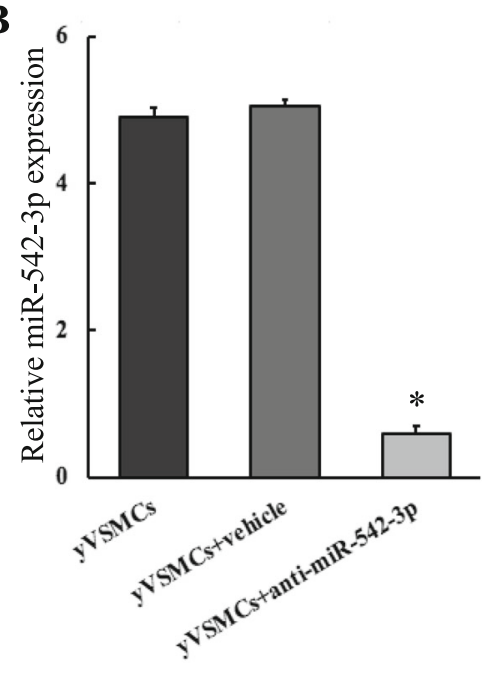

C

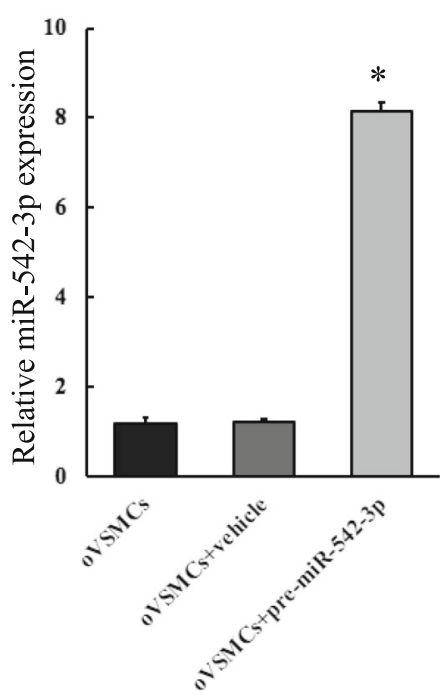

Fig. 1 miR-542-3p expression in yVMSCs and oVMSCs detected by qRT-PCR. a miR-542-3p expression was approximately downregulated 4-fold in oVSMCs comparing with yVSMCs. $n=3$. Versus yVSMCs, ${ }^{*} p<0.05$. b Transfection of anti-miR-542-3p into yVSMCs (yVSMCs+anti-miR-542-3p) significantly downregulated the miR-542-3p expression. $n=3$. Versus yVSMCs and yVSMCs transfected with its associated negative control (yVSMCs+vehicle) ${ }^{*} p<0.05$. c Transfection of pre-miR-542-3p into oVSMCs (oVSMCs+pre-miR-542-3p) significantly upregulated the miR-542-3p expression. $n=3$. Versus oVSMCs and oVSMCs transfected with its associated negative control (oVSMC+vehicle) ${ }^{*} p<0.05$

using lentivirus, and the miR-542-3p-expressing cells were cultured in growth medium containing $10 \mathrm{nM} \beta$-GP for 7 days to induce calcification. Western blot data showed that after $\beta$-GP induction, the expressions of the smooth muscle markers SM22 $\alpha$ and calponin were significantly higher in yVSMCs overexpressing miR-542-3p than those of yVSMCs (control) and yVSMCs infected with empty virus (vector) (Fig. 2a and b). After $\beta$-GP induction, the osteogenic markers OPN and Runx2 were expressed in control and vector yVSMCs. However, overexpressing miR-542-3p in yVSMCs decreased the OPN and Runx2 expressions induced by $\beta$-GP (Fig. 2a and b).

Calcium deposition assay by Alizarin red staining was performed to morphologically confirm osteogenic differentiation. After $\beta-$ GP induction, obvious calcium nodules were observed in control and vector yVSMCs, while in miR-542-3p-overexpressing yVSMCs, the calcium nodules were much fewer than in the other two groups (Fig. 2c). We further quantified the calcium deposition by eluting the Alizarin red and determining the optical density of the eluates. Consistent with the morphological data (Fig. 2c), these results demonstrated that the calcium deposition in miR-542-3p yVSMCs was suppressed when compared with that in control and vector yVSMCs (Fig. 2d). In general, these findings demonstrated that miR-542-3p overexpression could restrain the osteogenic phenotype of yVSMCs induced by $\beta$-GP. Thus, our results suggested that miR-542-3p might play a crucial role in regulating the osteogenic transition of VSMCs with senescence.

\section{miR-542-3p targeted BMP7}

BMPs were discovered by their capability to induce bone formation. To explore the potential targets of miR-542-3p involved in osteogenic transition of VSMCs, Targetscan and Miranda databases were used, and BMP7 was identified as a potential target. Therefore, BMP7 expression and its correlation with miR-542-3p were examined in yVSMCs induced by $\beta-G P$. As shown in Fig. 3a and b, after 7 days of induction by $\beta$-GP, yVSMCs overexpressing miR-542-3p expressed a significantly lower level of BMP7 than control yVSMCs and yVSMCs infected with empty virus, suggesting that miR-542-3p could suppress BMP7 


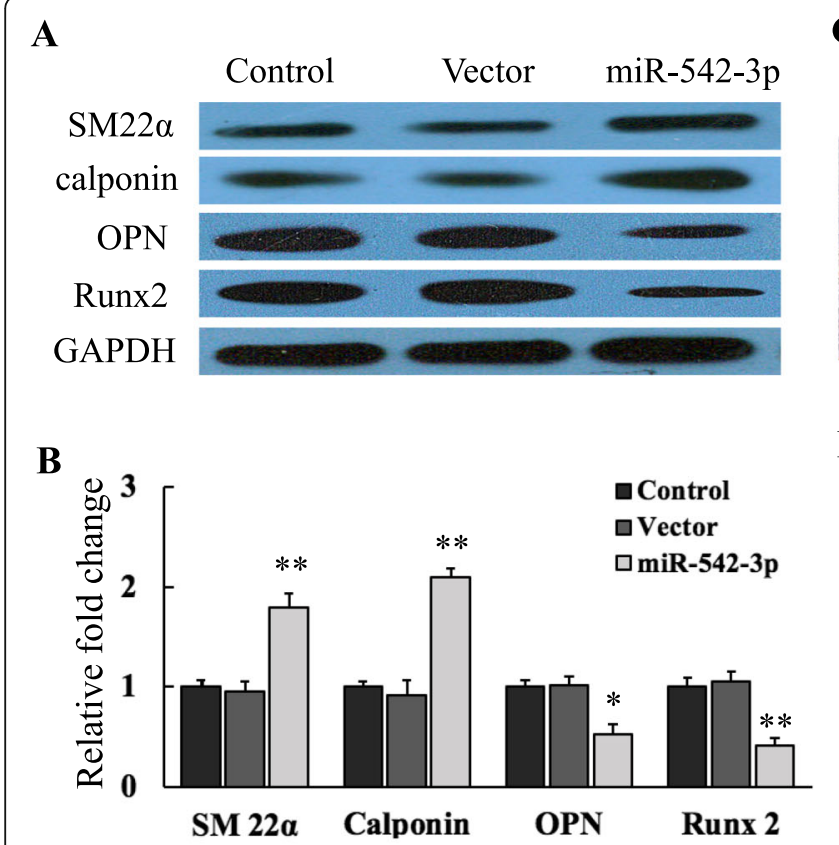

C

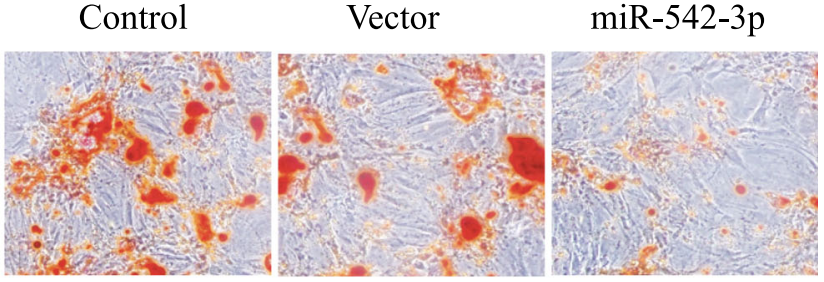

D

Fig. 2 miR-542-3p inhibited osteogenic transition in yVSMCs induced by $\beta$-GP. Control yVSMCs, yVSMCs infected with an empty virus vector, and yVSMCs stably expressing miR-542-3p (miR-542-3p) were induced to calcify by $\beta$-GP. a After 7 days of induction, expressions smooth muscle markers (SM22a and calponin) and osteogenic markers (OPN and Runx2) were investigated by Western blot. GAPDH was used as loading control. b Quantification of protein expressions by densitometric analysis. $n=3$. Versus control and vector yVSMCs, ${ }^{*} p<0.05$ and ${ }^{* *} p<0.01$. c After $\beta-G P$ induction for 7 days, calcium deposition was detected by Alizarin red staining. $\mathbf{d}$ Quantification of calcium deposition. $n=3$. Versus control and vector yVSMCs, ${ }^{* *} p<0.01$

expression in yVSMCs induced by $\beta-G P$, and that BMP7 is a potential target of miR-542-3p.

To confirm whether BMP7 is targeted by miR-542-3p, a luciferase reporter assay was performed using BMP7 3'-UTR constructs (Fig. 3c). The 3'-UTR of the luciferase reporter was generated containing BMP7 WT sequence or BMP7 MUT sequence. The luciferase assay showed that the overexpression of miR-542-3p strongly inhibited the luciferase activity in the BMP7 WT group but did not the BMP7 MUT group (Fig. 3d), which demonstrated that BMP7 is a direct target of miR-542-3p.

\section{miR-542-3p regulated osteogenic transition by targeting} BMP7

To explore whether the targeting of miR-542-3p to BMP7 regulating the osteogenic transition of VSMCs, yVSMCs stably overexpressing miR-542-3p (miRyVSMCs) were used for transduction with BMP7overexpressing lentivirus or control empty virus. The expression of smooth muscle markers SM22 $\alpha$ and calponin was significantly downregulated by overexpressing BMP7 in miR-yVSMCs, while the mRNA expression of osteogenic markers OPN and Runx2 was strongly increased in BMP7-overexpressing miR-yVSMCs when compared with that of the control miR-yVSMCs or miRyVSMCs transduced with control vector (Fig. 4a).
Consistent with the mRNA expression data, the Western blot results also demonstrated that overexpressing BMP7 in miR-yVSMCs decreased the protein expression levels of the smooth muscle markers SM22 $\alpha$ and calponin, and increased the protein expression levels of the osteogenic markers OPN and Runx2 (Fig. 4b and c).

Alizarin red staining was performed to examine calcium deposition. Calcium deposition was significantly higher in miR-yVSMCs overexpressing BMP7 compared with that of the control miR-yVSMCs or miR-yVSMCs transduced with control vector (Fig. $4 \mathrm{~d}$ and e). Together, these results demonstrated that overexpressing BMP7 in miR-yVSMCs reversed miR-542-3p's suppression of the osteogenic transition of $y \mathrm{VSMCs}$, suggesting that miR542-3p regulated osteogenic transition of VSMCs via targeting BMP7.

\section{Discussion}

In this study, by miRNA microarray, we identified 28 downregulated and 34 upregulated miRNAs in oVSMCs compared with yVSMCs. Among these miRNAs, miR542-3p, whose expression level was 14 folds downregulated in oVSMCs, was selected for further study. Our results showed that miR-542-3p suppressed the osteogenic differentiation of yVSMCs induced by $\beta-G P$. BMP7 was found to be directly targeted by miR-542-3p, and 


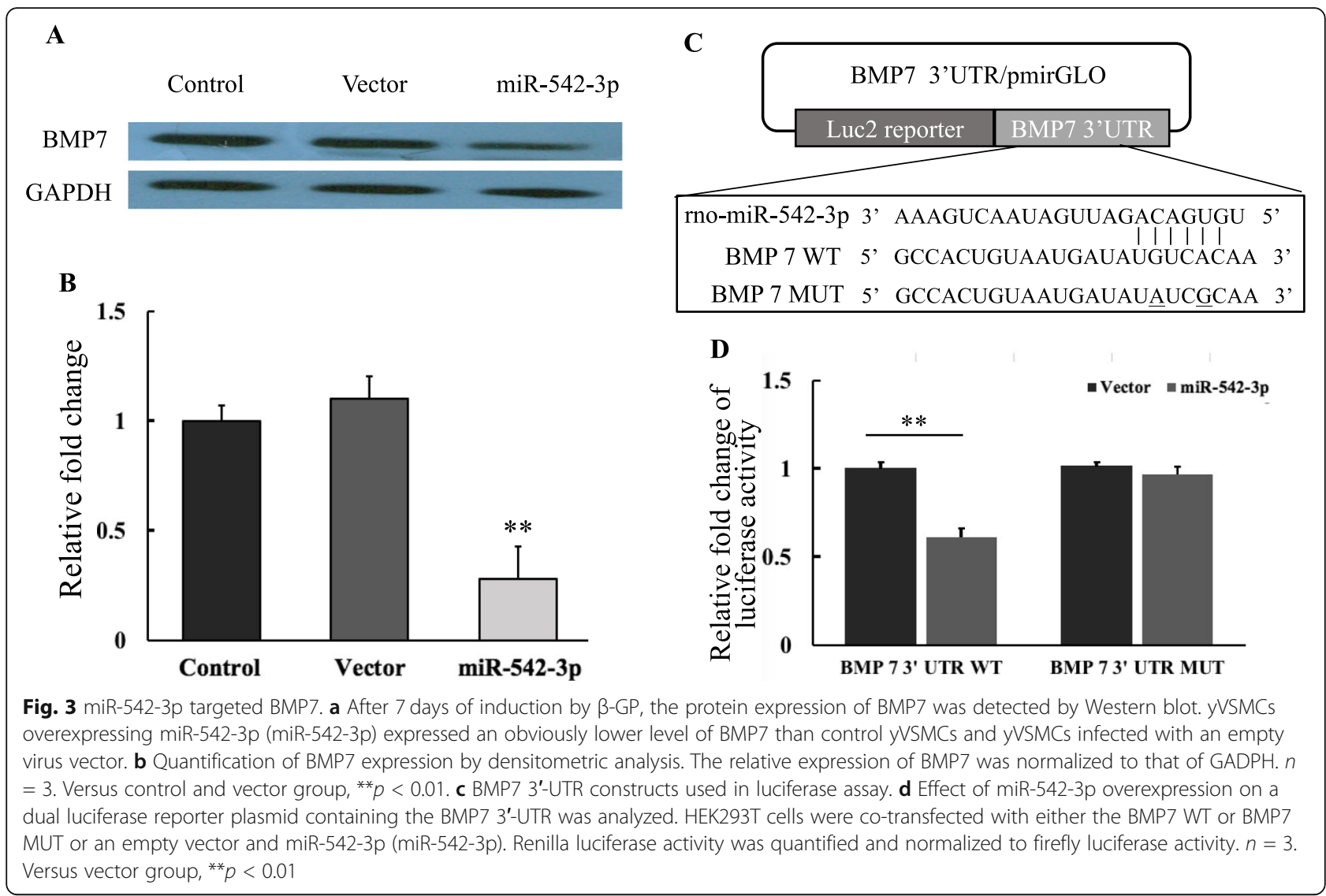

overexpressing BMP7 in miR-542-3p-expressing yVSMCs could reverse miR-542-3p's inhibition of osteogenic differentiation. Our findings indicated that downregulation of miR-542-3p promotes the osteogenic transition of VSMCs in the aging rat via targeting BMP7.

Cardiovascular disease is a major cause of death worldwide and the biological mechanisms of its development remain unclear. In recent years, the roles of miRNAs in regulating cell functions have been proved in the cardiovascular system, such as cardiomyocytes, endothelial cells, fibroblasts, and smooth muscle cells [33]. miRNAs are noncoding RNAs that inhibit protein translation and/or anneal to mRNAs and promote their cleavage. Accumulating reports have proved the involvement of miRNAs in pathological changes of the cardiovascular system [1, 12, 33].

Cardiovascular diseases are a prevailing issue in aging patients. Vascular senescence has a close connection with a broad spectrum of cardiovascular diseases, with the characteristics of endothelial dysfunction and phenotypic transition of smooth muscle cells, resulting in increased vascular stiffness and increased thickness of vascular walls. It has been reported that the ageassociated phenotypic transition of VSMCs is a crucial contributor to vascular remodeling [17, 25]. However, the mechanism that drives phenotypic transition of
VSMCs with aging remains unclarified. In this study, using RNAs extracted from the in vitro cultured VSMCs, we explored the miRNA expression profile by microarray. Owing that rat aortas are composed of many kinds of cells, including VSMCs, endothelial cells, and fibroblasts, to avoid the contamination of RNA from endothelial cells and fibroblasts, we extracted the RNAs extracted from the in vitro cultured VSMCs instead from the aortas, which may be a drawback of our study. By microarray, we investigated miRNA expressions in yVSMCs and oVSMCs and identified 28 miRNAs downregulated in oVSMCs, including miR-196a-5p $(\sim 107-$ fold), miR-196b-5p ( 48-fold), miR-542-3p ( 14-fold), and miR-363-5p ( 8-fold). miR-196a-5p and miR-196b$5 \mathrm{p}$ were reported to be involved in regulating cancer progression [26, 27, 36, 37]. Thus, miR-542-3p was selected in the present study.

miR-542-3p was previously demonstrated to be involved in osteoblast proliferation and differentiation [14]; however, its function in vascular cells is unclear. In our study, inhibited expression of osteogenic markers and calcium deposition were observed in miR-542-3poverexpressing yVSMCs, while the expression of smooth muscle markers was upregulated, indicating a pivotal role of miR-542-3p in regulating osteogenic transition of 


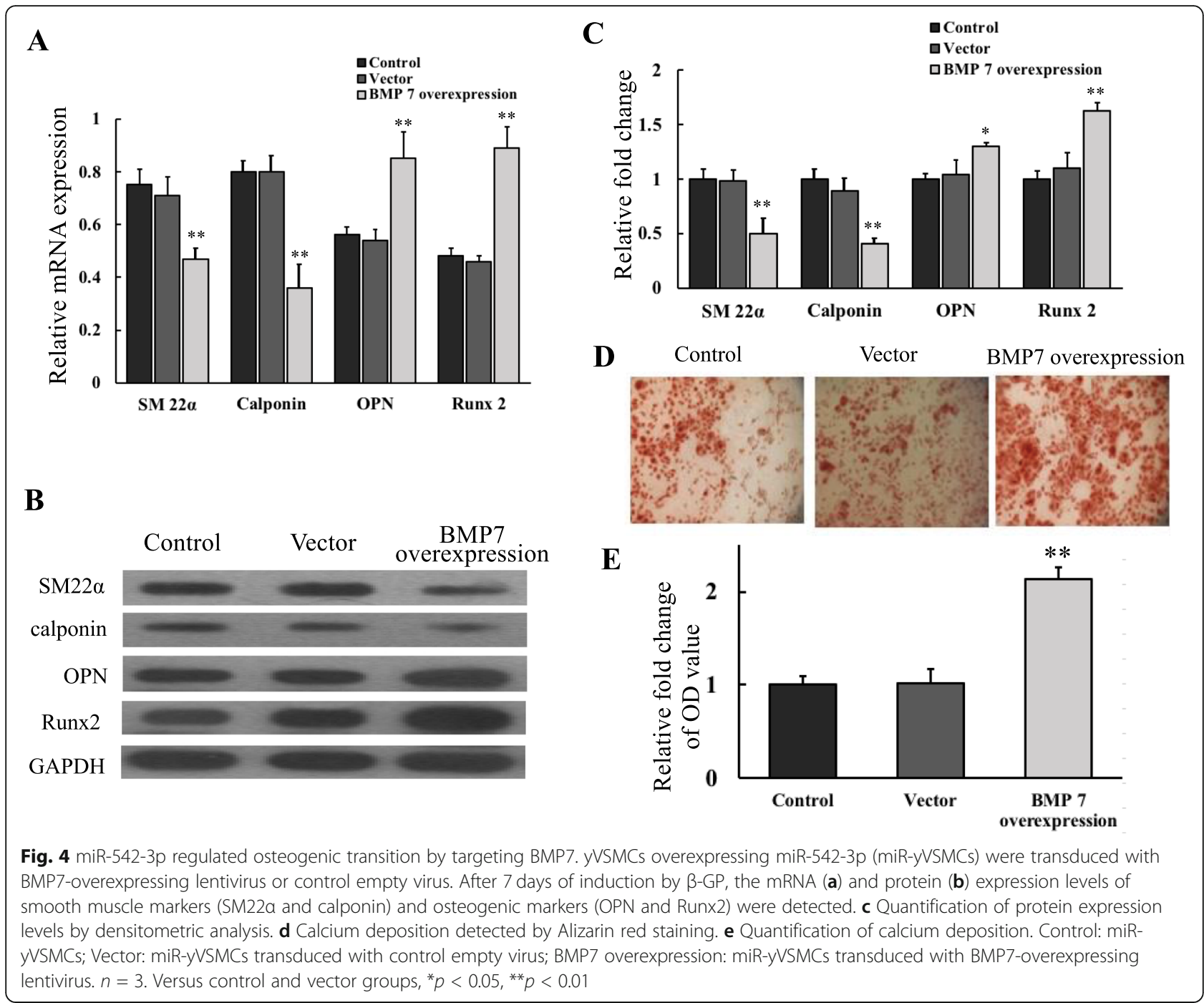

VSMCs. Certain miRNAs have been reported to induce switching of VSMCs to the synthetic phenotype, including miR-let-7 g [32], miR-132 [5], miR-145 [19], miR-32 [20, 21], and miR-22 [36, 37]; some of which, for example miR-145 and miR-32, were identified to regulate osteogenic switching of VSMCs. However, miRNAs involved in the age-associated phenotypic conversion of VSMCs have not been completely identified. miR-542$3 p$ has been proved to suppress cancer cell growth [20, $21,30]$. Our previous work showed that the downregulation of miR-542-3p promoted neointimal formation in aging rats [28]. In the present study, we showed that miR-542-3p regulates the osteogenic transition of VSMCs, and with aging, downregulated miR-542-3p was associated with the calcification of the vascular wall.

BMPs are a group of proteins originally discovered to have the capability in inducing osteogenesis and chondrogenesis. There are more than 20 members in BMP family, and some family members, especially BMP2 and BMP7, have been shown to induce osteoblast differentiation and bone formation in vivo and ex vivo [35]. BMP signaling was also found to be associated with vascular diseases [4, 8] and vascular calcification [11, 38]. In our study, BMP7 was confirmed to be a target of miR-542-3p. Consistent with our study, it was reported that miR-542-3p suppresses osteoblast cell proliferation and differentiation by targeting BMP7 signaling [14]. The miRNAs miR-22 [22] and miR-22-3p [34] were also demonstrated to target BMP7. Additionally, using an online database miRDB (http://mirdb.org), we predicted the potential targets of rno-miR-542-3p, and identified 328 predicted targets for miR-542-3p (Additional file 1: Table S3), which could further help us to understand the role of miR-542-3p in VSMCs.

Generally, in this study, overexpression of miR-542-3p inhibited BMP7 expression in yVSMCs and osteogenic differentiation of yVSMCs, which can be reversed by overexpressing BMP7 in miR-542-3p-overexpressing 


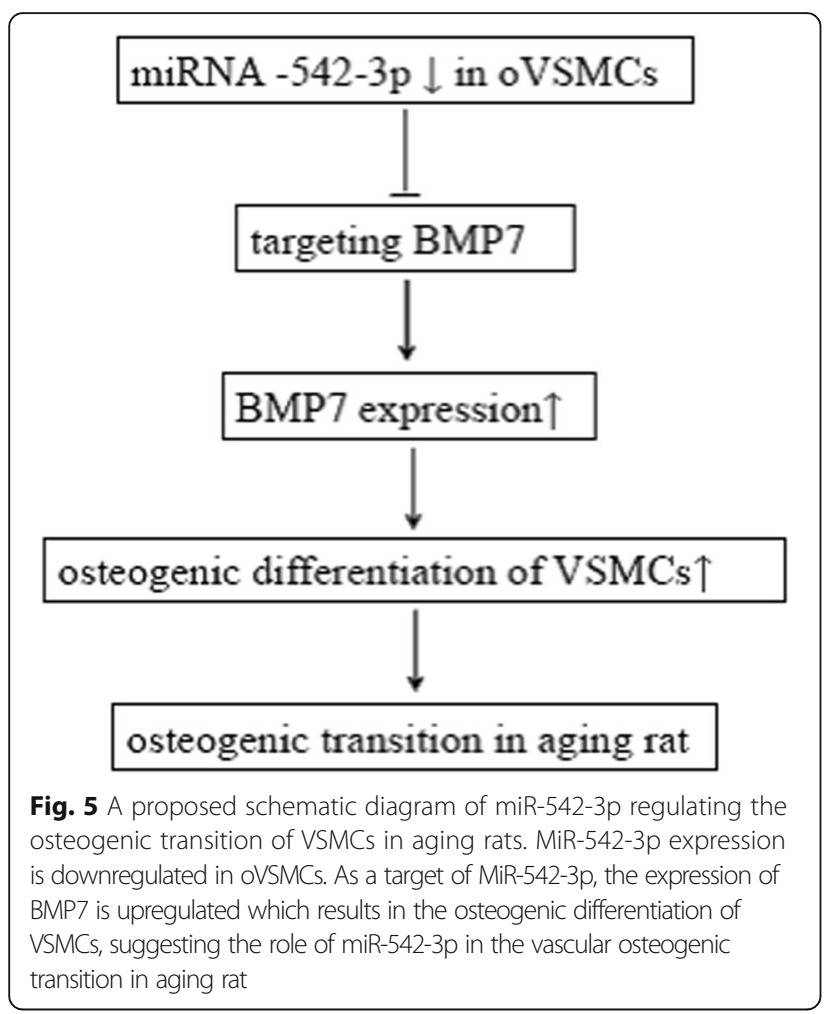

yVSMCs, suggesting that miR-542-3p regulates osteogenic transition of VSMCs via targeting BMP7. BMP7 is extensively expressed in various tissues and is connected to the development and pathological changes of bone, renal, and ocular systems, as well as the central nervous system [15]. Although BMP7 has been demonstrated to play important role in osteogenesis [35], its role in the osteogenic transition of VSMCs and vascular calcification has not been well documented. Instead, some studies related BMP2 to vascular calcification by its role in regulating osteoblast differentiation of VSMCs $[6,18$, 39]. Thus, our study demonstrated novel signaling of BMP7, regulating the osteogenic transition of VSMCs and vascular calcification.

\section{Conclusions}

We demonstrated that miR-542-3p was differentially expressed in oVSMCs and yVSMCs and demonstrated that miR-542-3p overexpression prohibited BMP7 expression and osteogenic differentiation in yVSMCs induced by $\beta$-GP, which can be reversed by overexpressing BMP7 in miR-542-3p-overexpressing yVSMCs. A proposed schematic diagram of miR-542-3p regulating the osteogenic transition of VSMCs in aging rats is shown in Fig. 5. Together, our results suggested that miR-542-3p regulates the osteogenic transition of VSMCs in aging rats by targeting BMP7. These findings are helpful for a better understanding of the role of miRNAs in regulating the osteogenic transition of VSMCs in aging.

\section{Supplementary information}

Supplementary information accompanies this paper at https://doi.org/10. 1186/s40246-019-0245-z.

Additional file 1: Table S1. oVSMCs vs yVSMCs 2.0 fold upregulated miRNAs. Table S2. oVSMCs vs yVSMCs 2.0 fold downregulated miRNAs. Table S3. Predicted targets of miR-542-3p.

Acknowledgements

Not applicable.

\section{Authors' contributions}

$\mathrm{HL}$ performed the experiment and wrote the manuscript. HW analyzed the data. SY designed the study. HL and DQ funded the study. All authors read and approved the final manuscript.

\section{Funding}

This work was supported by grants from the Natural National Science Foundation of China (No. 81601232), Chongqing Research Program of Basic Research and Frontier Technology (No. cstc2016jcyjA1637), Special Fund for High-level Talents (Chen Chen Team) of the People's Government of Luzhou-Southwestern Medical University, and supported by Sichuan Science and Technology Program (No. 2018JY0402).

Availability of data and materials

Not applicable.

Ethics approval and consent to participate

Not applicable.

\section{Consent for publication}

Not applicable.

\section{Competing interests}

The authors declare that they have no competing interests.

\section{Author details}

${ }^{1}$ Department of Cardiology, Second Hospital Affiliated to the Army Medical University, Xinqiao Hospital, Chongqing 400037, China. ${ }^{2}$ The Precision Medicine Institute, The Third Affiliated Hospital, Southern Medical University, Guangzhou 510150, Guangdong, China. ${ }^{3}$ Department of Orthopaedics, The Second Affiliated Hospital of Southwest Medical University, Lu Zhou 646000, Sichuan, China. ${ }^{4}$ Department of Cardiology and Neurology, The Second Affiliated Hospital of Southwest Medical University, 184 Chunhui Street, Lu Zhou 646000, Sichuan, China. ${ }^{5}$ Department of Orthopedics, General Hospital of Shenyang Military Area Command of Chinese PLA, Shenyang 110016, Liaoning, China.

Received: 16 June 2019 Accepted: 17 October 2019 Published online: 11 December 2019

\section{References}

1. Barwari T, Joshi A, Mayr M. MicroRNAs in cardiovascular disease. Journal of the American College of Cardiology. 2016;68(23):2577-84. https://doi.org/10. 1016/j.jacc.2016.09.945.

2. Bronze-Da-Rocha E. MicroRNAs expression profiles in cardiovascular diseases. BioMed Research International. 2014;2014:985408. https://doi.org/ 10.1155/2014/985408.

3. Brott TG, Hobson RW, Howard G, Roubin GS, Clark WM, Brooks W, Meschia JF. Stenting versus endarterectomy for treatment of carotid-artery stenosis. New England J Med. 2010;363(1):11-23. https://doi.org/10.1056/ NEJMoa0912321.

4. Cai J, Pardali E, Sánchez-Duffhues G, Ten Dijke P. BMP signaling in vascular diseases. FEBS Letters. 2012;586:1993-2002. https://doi.org/10.1016/j.febslet. 2012.04.030

5. Chen WJ, Chen YH, Hsu YJ, Lin KH, Yeh YH. MicroRNA-132 targeting PTEN contributes to cilostazol-promoted vascular smooth muscle cell differentiation. Atherosclerosis. 2018:274:107. https://doi.org/10.1016/j. atherosclerosis.2018.04.030. 
6. Davenport C, Harper E, Forde H, Rochfort KD, Murphy RP, Smith D, Cummins PM. RANKL promotes osteoblastic activity in vascular smooth muscle cells by upregulating endothelial BMP-2 release. Int J Biochem Cell Biol. 2016;77(Part A):171-80. https://doi.org/10.1016/j.biocel.2016.06.009.

7. Durham AL, Speer MY, Scatena M, Giachelli CM, Shanahan CM. Role of smooth muscle cells in vascular calcification: implications in atherosclerosis and arterial stiffness. Cardiovasc Res. 2018;114(4):590-600. https://doi.org/10. 1093/cvr/cvy010.

8. García de Vinuesa A, Abdelilah-Seyfried S, Zwijsen A, Bailly S. BMP signaling in vascular biology and dysfunction. Cytokine Growth Factor Rev. 2016;27: 65-79. https://doi.org/10.1016/j.cytogfr.2015.12.005.

9. Gregory CA, Gunn WG, Peister A, Prockop DJ. An Alizarin red-based assay of mineralization by adherent cells in culture: comparison with cetylpyridinium chloride extraction. Analytical Biochemistry. 2004;329(1):77-84. https://doi. org/10.1016/j.ab.2004.02.002

10. Hobson RW, Howard VJ, Roubin GS, Brott TG, Ferguson RD, Popma J, Howard G. Carotid artery stenting is associated with increased complications in octogenarians: 30-Day stroke and death rates in the CREST lead-in phase. J Vasc Surg. 2004;40(6):1106-11. https://doi.org/10.1016/j.jvs. 2004.10.022

11. Hruska KA, Mathew S, Saab G. Bone morphogenetic proteins in vascular calcification. Circulation Research. 2005;97(2):105-14. https://doi.org/10.1161/ 01.RES.00000175571.53833.6c

12. Islas J, Moreno-Cuevas J. A microRNA perspective on cardiovascular development and diseases: an update. Int J Mol Sci. 2018;19(7):2075. https:// doi.org/10.3390/ijms19072075.

13. Ito T, Yagi S, Yamakuchi M. MicroRNA-34a regulation of endothelial senescence. Biochemical and Biophysical Research Communications. 2010; 398(4):735-40. https://doi.org/10.1016/j.bbrc.2010.07.012.

14. Kureel J, Dixit M, Tyagi AM, Mansoori MN, Srivastava K, Raghuvanshi A, Singh D. MiR-542-3p suppresses osteoblast cell proliferation and differentiation, targets BMP-7 signaling and inhibits bone formation. Cell Death Dis. 2014;5(2):e1050-11. https://doi.org/10.1038/cddis.2014.4.

15. Kusakawa Y, Mikawa S, Sato K. BMP7 expression in the adult rat brain. IBRO Reports. 2017;3:72-86. https://doi.org/10.1016/j.ibror.2017.06.002.

16. Lacolley P, Regnault V, Avolio AP. Smooth muscle cell and arterial aging: basic and clinical aspects. Cardiovascular Research. 2018;114(4):513-28. https://doi.org/10.1093/cvr/cvy009.

17. Lacolley P, Regnault V, Nicoletti A, Li Z, Michel JB. The vascular smooth muscle cell in arterial pathology: a cell that can take on multiple roles. Cardiovasc Res. 2012;59(2):1171-7. https://doi.org/10.1093/cvr/cvs135.

18. Li $X$, Yang H-Y, Giachelli CM. BMP-2 promotes phosphate uptake, phenotypic modulation, and calcification of human vascular smooth muscle cells. Atherosclerosis. 2008;199(2):271. https://doi.org/10.1016/j. atherosclerosis.2007.11.031

19. Liao J, Zhang Y, Wu Y, Zeng F, Shi L. Akt modulation by miR-145 during exercise-induced VSMC phenotypic switching in hypertension. Life Sciences. 2018;199:71-9. https://doi.org/10.1016/j.lfs.2018.03.011.

20. Liu B, Li J, Zheng M, Ge J, Li J, Yu P. MiR-542-3p exerts tumor suppressive functions in non-small cell lung cancer cells by upregulating FTSJ2. Life Sci. 2017a;188(1):87-95. https://doi.org/10.1016/j.lfs.2017.08.018.

21. Liu J, Xiao X, Shen Y, Chen L, Xu C, Zhao H, Zu X. MicroRNA-32 promotes calcification in vascular smooth muscle cells: implications as a novel marker for coronary artery calcification. PLoS ONE. 2017b;12(3):e0174138. https://doi. org/10.1371/journal.pone.0174138.

22. Long J, Badal SS, Wang Y, Chang BHJ, Rodriguez A, Danesh FR. MicroRNA22 is a master regulator of bone morphogenetic protein-7/6 homeostasis in the kidney. J Biol Chem. 2013;288(51):36202-14. https://doi.org/10.1074/jbc. M113.498634

23. Magenta A, Cencioni C, Fasanaro P, Zaccagnini G, Greco S, Sarra-Ferraris G, Capogrossi MC. MiR-200C is upregulated by oxidative stress and induces endothelial cell apoptosis and senescence via ZEB1 inhibition. Cell Death Differ. 2011;18:1628-39. https://doi.org/10.1038/cdd.2011.42.

24. Mukamal KJ, Kronmal RA, Tracy RP, Cushman M, Siscovick DS. Traditional and novel risk factors in older adults: cardiovascular risk assessment late in life. Am J Geriatr Cardiol. 2004;13(2):69-80.

25. Owens GK, Kumar MS, Wamhoff BR. Molecular regulation of vascular smooth muscle cell differentiation in development and disease. Physiol Rev. 2004;84(3):194-204. https://doi.org/10.1152/physrev.00041.2003.

26. Pan J, Li X, Wu W, Xue M, Hou H, Zhai W, Chen W. Long non-coding RNA UCA1 promotes cisplatin/gemcitabine resistance through CREB modulating
miR-196a-5p in bladder cancer cells. Cancer Lett. 2016;383(1):64-76. https:// doi.org/10.1016/j.canlet.2016.08.015.

27. Porretti J, Dalton GN, Massillo C, Scalise GD, Farré PL, Elble R, De Siervi A. CLCA2 epigenetic regulation by CTBP1, HDACs, ZEB1, EP300 and miR-196b$5 p$ impacts prostate cancer cell adhesion and EMT in metabolic syndrome disease. Int J Cancer. 2018;143(4):897-906. https://doi.org/10.1002/ijc.31379.

28. Qian DH, Gao P, Feng H, Qin ZX, Li JB, Huang L. Down-regulation of mir$542-3 p$ promotes neointimal formation in the aging rat. Vasc Pharmacol. 2015:72:118-29. https://doi.org/10.1016/j.vph.2015.05.010.

29. Roubin GS, New G, lyer SS, Vitek JJ, Al-Mubarak N, Liu MW, Kuntz RE. Immediate and late clinical outcomes of carotid artery stenting in patients with symptomatic and asymptomatic carotid artery stenosis: a 5-year prospective analysis. Circulation. 2001;103(4):532-7. https://doi.org/10.1161/ 01.CIR.103.4.532

30. Tao J, Liu Z, Wang Y, Wang L, Yao B, Li Q, Liu Q. MiR-542-3p inhibits metastasis and epithelial-mesenchymal transition of hepatocellular carcinoma by targeting UBE3C. Biomed Pharmacother. 2017;93:420-8. https://doi.org/10.1016/j.biopha.2017.06.070.

31. Vallot $O$, Combettes $L$, Jourdon $P$, Inamo J, Marty I, Claret M, Lompré AM. Intracellular $\mathrm{Ca}(2+)$ handling in vascular smooth muscle cells is affected by proliferation. Arterioscler Thromb Vasc Biol. 2000;20(5):1225-35. https://doi. org/10.1161/01.ATV.20.5.1225.

32. Wang TM, Chen KC, Hsu PY, Lin HF, Wang YS, Chen CY, Juo SHH. microRNA let-7g suppresses PDGF-induced conversion of vascular smooth muscle cell into the synthetic phenotype. J Cell Mol Med. 2017;21(12):3592-601. https:// doi.org/10.1111/jcmm.13269.

33. Wojciechowska A, Osiak A, Kozar-Kamińska K. MicroRNA in cardiovascular biology and disease. Adv Clin Exp Med. 2017;26(5):868-74. https://doi.org/ 10.17219/acem/62915.

34. Wu C, Zheng Z, Ren W, Deng T, Li Y, Yang L, Guo L. Mm9_circ 009056 enhances osteogenesis by targeting BMP7 via CGRP-mediated miR-22-3p. Biochemical and Biophysical Research Communications. 2018;501(1):199205. https://doi.org/10.1016/j.bbrc.2018.04.215

35. Yamaguchi A, Sakamoto K, Minamizato T, Katsube K, Nakanishi S. Regulation of osteoblast differentiation mediated by BMP, Notch, and CCN3/NOV. Japanese Dent Sci Rev. 2008;44(1):48-56. https://doi.org/10.1016/j.jdsr.2007. 11.003.

36. Yang F, Chen Q, He S, Yang M, Maguire EM, An W, Xiao Q. miRNA-22 is a novel mediator of vascular smooth muscle cell phenotypic modulation and neointima formation. Circulation. 2017a;137(17):1824-41. https://doi.org/10. 1161/CIRCULATIONAHA.117.027799.

37. Yang JP, Yang JK, Li C, Cui ZQ, Liu HJ, Sun XF, Jiao BH. Downregulation of ZMYND11 induced by miR-196a-5p promotes the progression and growth of GBM. Biochem Biophys Res Commun. 2017b;494(3-4):674-80. https://doi. org/10.1016/j.bbrc.2017.10.098

38. Yao Y, Bennett BJ, Wang X, Rosenfeld ME, Giachelli C, Lusis AJ, Boström KI. Inhibition of bone morphogenetic proteins protects against atherosclerosis and vascular calcification. Circulation Research. 2010;107(4):485-94. https:// doi.org/10.1161/CIRCRESAHA.110.219071.

39. Zhao YG, Meng FX, Li BW, Sheng YM, Liu MM, Wang B, Xiu RJ. Gelatinases promote calcification of vascular smooth muscle cells by up-regulating bone morphogenetic protein-2. Biochem Biophys Res Commun. 2016; 470(2):287-93. https://doi.org/10.1016/j.bbrc.2016.01.067.

40. Zhou J, Wang K-C, Wu W, Subramaniam S, Shyy JY-J, Chiu J-J, Chien S. MicroRNA-21 targets peroxisome proliferators-activated receptor- in an autoregulatory loop to modulate flow-induced endothelial inflammation. Proc Natl Acad Scinces. 2011;108(25):10355-60. https://doi.org/10.1073/pnas. 1107052108.

\section{Publisher's Note}

Springer Nature remains neutral with regard to jurisdictional claims in published maps and institutional affiliations. 